\title{
Atividades de coleta e origem floral do pólen armazenado em colônias de Plebeia saiqui (Holmberg) (Hymenoptera, Apidae, Meliponinae) no sul do Brasil
}

\author{
Raquel A. Pick 1, 2 \\ Betina Blochtein ${ }^{1}$
}

\begin{abstract}
Collection activities and floral origin of the stored pollen in colonies of Plebeia saiqui (Holmberg) (Hymenoptera, Apidae, Meliponinae) in south Brazil. Four colonies of Plebeia saiqui (Holmberg, 1903), of São Francisco de Paula, were studied during the period from October/1998 to October/1999. The counting of the bees was proceeded monthly, differentiated workers that came back with and without pollen in the corbicula. Grains of pollen of pots previously marked were collected monthly and identified. The percentage of the pollen types of the samples was estimated: $20 \%$ of Asteraceae, 17\% Myrtaceae, 15\% type Meliaceae and 10\% Euphorbiaceae. The remaining corresponds to other pollen types of small representation, besides those the were no identified. The climatic influence on the pollen collection was analyzed being used simple and multiple regressions. It was verified that in the spring and in the summer the temperature, the solar irradiation and relative humidity were significant for the pollen foraging. During autumn and winter the relative humidity had smaller influence in the pollen collection.

KEY WORDS. Plebeia, stingless bees, pollen analysis, foraging, climatic influence
\end{abstract}

Os meliponíneos são visitantes florais por excelência, devido ao hábito alimentar e à diversificação morfofuncional associada a um grande número de espécies vegetais (RAMALHO et al. 1991). São responsáveis por 40 a $90 \%$ da polinização das árvores nativas (KERR 1996).

Os meliponíneos desempenham atividades de vôo visando obter materiais para construção do ninho, eliminar dejetos e principalmente para coletar alimento para a colônia. A dieta é composta de proteínas, pólen e ocasionalmente esporos de fungos, e de carboidratos obtidos do néctar (ABSY \& KERR 1977; FREE \& WILLIAMS 1977).

Pesquisas sobre atividades de vôo de representantes de Meliponinae relacionadas aos parâmetros meteorológicos, foram desenvolvidas com Tetragonisca angustula (Latreille, 1811) (IWAMA 1977), Plebeia emerina (Friese, 1900) (KLEINERT-GIOVANNINI 1982) e Plebeia remota (Holmberg, 1903) (IMPERATRIZ-FONSECA et al. 1985).

Em São Paulo foram desenvolvidos estudos sobre dieta floral de Meliponinae em T. angustula (IWAMA \& MELHEM 1979; IMPERATRIZ-FONSECA et al. 1984; P. remota (RAMALHO et al. 1985), P. saiqui (Holmberg, 1903) (KLEINERT-GIOVANNINI et al. 1987; PIRANI \& CORTOPASSI-LAURINO 1994), Scaptrotrigona bipunctata (Lepeletier, 1836), Scaptrotrigona depilis (Moure, 1942), Scaptrotrigona postica

\footnotetext{
1) Faculdade de Biociências, Pontifícia Universidade Católica do Rio Grande do Sul. Avenida Ipiranga 6681, 90619-900 Porto Alegre, Rio Grande do Sul, Brasil.

2) Bolsista da CAPES.
} 
(Latreille, 1807) (RAMALHo 1990). No Rio Grande do Sul ainda não foram desenvolvidos estudos a respeito de coleta de alimentos e do pólen armazenado em ninhos de Meliponinae.

Plebeia saiqui é conhecida popularmente como abelha mirim, devido ao seu pequeno tamanho $(4 \mathrm{~mm})$. Nidifica em troncos ocos e apresenta colônias com até 7.000 indivíduos. São encontradas no Paraná, Santa Catarina e Rio Grande do Sul (KLeinerT-Giovannini et al. 1987; WitTMANn \& HofFMAN 1990; PiRANi \& CORTOPASSI-LAURINO 1994). Plebeia saiqui é freqüentemente encontrada em São Francisco de Paula, Rio Grande do Sul, onde foi registrada por WILMS et al. (1997).

O conhecimento das fontes de pólen utilizadas por esse meliponíneo permitirá avaliar o comportamento das forrageiras, compreender melhor a utilização de recursos florais, além de destacar a importância de plantas para a manutenção dessas abelhas.

O presente trabalho tem como objetivos avaliar a atividade de coleta de pólen por operárias e identificar a origem floral do pólen armazenado em ninhos de $P$. saiqui, no município de São Francisco de Paula.

\section{MATERIAL E MÉTODOS}

\section{Área de estudo}

O estudo foi desenvolvido durante o período de outubro de 1998 a setembro de 1999, no Centro de Pesquisas e Conservação da Natureza Pró-Mata (CPCN Pró-Mata/PUCRS), situado no município de São Francisco de Paula, Rio Grande

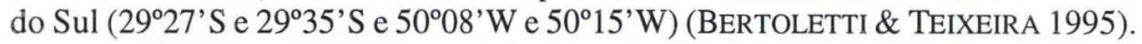

A vegetação predominante na área do $\mathrm{CPCN}$ Pró-Mata é constituída pela Floresta com Araucária, Mata Atlântica e campos. Apesar desses ecossistemas apresentarem-se ameaçados devido a agropecuária, encontram-se ainda no estrato dominante a Araucaria angustifolia (Bert.) O. Kuntze (Araucariaceae) e espécies florísticas das famílias Lauraceae, Cryptocarya aschersoniana Mez (canela-areia), Ocotea pulchella Mart. (canela langeana), Ocotea puberula Nees (canela-sebo), Rosaceae, Prunus sellowii Koehne (pessegueiro-bravo) e Leguminosae, Mimosa scabrella Benth. (bracatinga) (TEIXEIRA \& COURA-NETO 1986; BERTOLETTI \& TEIXEIRA 1995).

A maior parte da área encontra-se situada entre 600 e $900 \mathrm{~m}$ de altitude. $\mathrm{O}$ clima está classificado como superúmido a úmido, com regime pluviométrico oscilando entre 1750 e $2500 \mathrm{~mm}$ anuais. A temperatura média anual é de $14,5^{\circ} \mathrm{C}$, sendo as médias anuais mínimas e máximas, respectivamente, $9,9^{\circ} \mathrm{C}$ e $20,3^{\circ} \mathrm{C}$ (IBGE 1986; BERTOLETTI \& TEIXEIRA 1995).

Quatro colônias de $P$. saiqui (A, D, E e F), do mesmo município, foram transferidas para caixas de madeira com $3 \mathrm{~cm}$ de espessura $(37 \times 18 \times 28 \mathrm{~cm})$. As colônias A e D, foram instaladas no laboratório e as colônias E e F, no meliponário do CPCN Pró-Mata. As duas primeiras foram selecionadas para observação da atividade de coleta de pólen e as demais para análise do pólen armazenado em potes.

\section{Atividade de coleta de pólen}

As atividades de vôo das abelhas das colônias A e D foram registradas em mensalmente, durante o período de outubro de 1998 a setembro de 1999. As 
operárias que entravam com pólen nas corbículas foram quantificadas com auxílio de contador manual durante 10 minutos, a cada hora, conforme descrito por OLIVEIRA (1973). Essa atividade foi realizada junto a entrada do ninho do início até o término das atividades de vôo, totalizando 175 observações.

\section{Análise do pólen}

O pólen armazenado nas colônias E e F, foi coletado no período de novembro/1998 a outubro/1999. Potes de pólen que estavam sendo aprovisionados foram marcados com palitos de madeira. Mensalmente, amostrou-se de 2-4 g de pólen de 1-6 potes de pólen fechados, extraindo-se o conteúdo com espátula, desde o ápice até a base do pote.

A partir de cada amostra de pólen foram confeccionadas três preparações, coradas com fucsina e não coradas, de acordo com o método proposto por WoDEHOUSE (1935).

A identificação dos grãos de pólen foi feita consultando-se literatura especializada (SALGADO-LABOURIAU 1973; GRONQUIST 1981; HoOGHIEMSTRA 1984; FAEGRI \& IVERSEN 1989; ROUBIK \& MORENO 1991; HERREIRA \& URREGO 1996) e por comparação com lâminas da Palinoteca de Referência do Laboratório de Pesquisas Biológicas (LPB) da PUCRS. Os grãos de pólen foram identificados a nível de família, e quando possível, de gênero e espécie. Contudo, em alguns casos, devido a homogeneidade polínica de alguns grupos (gêneros, famílias), a identificação ficou limitada a nível de "tipo polínico" (categoria que agrupa grãos de pólen com um conjunto de características semelhantes a uma espécie, gênero ou família).

A representação percentual dos tipos polínicos foi estimada contando-se 1000 grãos de pólen, ao acaso, em três lâminas de cada amostra (VERGERON 1964).

\section{Influência dos parâmetros meteorológicos na coleta de pólen}

Os parâmetros meteorológicos (temperatura, umidade relativa, vento e irradiação solar) foram obtidos em intervalos de 30 minutos, por meio da estação meteorológica SEBA (versão 1.50, Rel. 009) instalada a aproximadamente $3 \mathrm{~km}$ das colônias. Os dados foram coletados entre 6:30 e 19:30 h e analisados nos períodos de outubro, novembro e dezembro (postura) e maio, junho e julho (diapausa).

A influência dos parâmetros meteorológicos sobre a coleta de pólen pelas abelhas foi analisada utilizando-se regressão simples para verificar a significância de cada fator isoladamente. Com a regressão múltipla buscou-se relacionar os fatores que contribuíram significativamente para alterações dessa atividade. Os dados foram plotados por dia e de forma agrupada, isto é, sazonal, visando comparar os padrões.

\section{RESULTADOS E DISCUSSÃO}

\section{Atividades de coleta de pólen}

Nas colônias A e D, verificou-se que a atividade de coleta de pólen foi distinta de outubro a dezembro/1998 (postura) e maio a julho/1999 (diapausa). Durante os meses mais quentes concentrou-se o maior esforço de coleta de pólen registrado ao longo do ano. A partir de fevereiro/1999, essa atividade diminuiu gradativamente, tornando-se menos intensa (Fig. 1). 

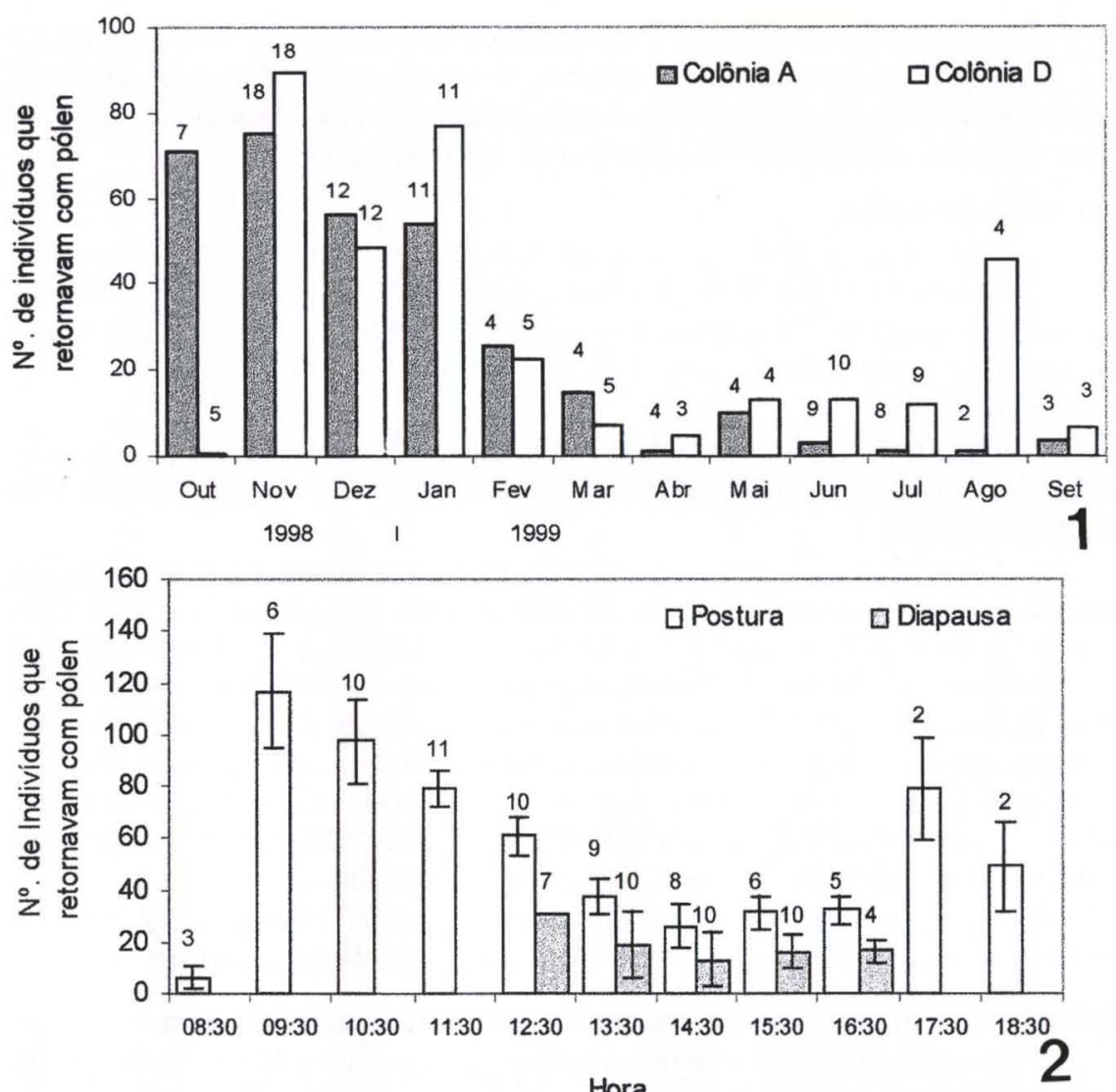

Hora

Figs 1-2. (1) Média do número de abelhas de $P$. saiqui, que realizavam atividades de vôo ao longo de um ano; os números acima das colunas indicam o total de observações; (2) média e desvio padrão do número de indivíduos de duas colônias de $P$. saiqui que retornavam com pólen, em relação ao horário, durante os períodos de postura e diapausa; os números acima das colunas indicam o total de observações.

Observou-se um aumento significativo de entrada de pólen na colônia D, em agosto, imediatamente após o reinicio da postura pela rainha (26/VII/1999). Por outro lado, não se registrou um aumento importante de entrada de pólen na colônia A. Neste caso a oviposição iniciou em 22/VIII/1999.

No que diz respeito a amplitude diária de forrageamento de pólen, verificouse que, durante o período de postura, variou entre 8:30 e 18:30 h, apresentando maior intensidade no início da manhã em torno das 9:30 h. Na diapausa, o período de coleta de pólen foi reduzido, iniciando às $12: 30 \mathrm{~h}$ e terminando às $16: 30 \mathrm{~h}$. Ocorreu uma redução de seis horas diárias de coleta de pólen, em relação ao período de postura, totalizando quatro horas (Fig. 2). 
ROUBIK (1989), atribuiu o pico matutino de coleta de pólen à disponibilidade do recurso. Pólen é comumente abundante e disponível nas primeiras horas da manhã, como verificado para $P$. saiqui, mas torna-se escasso à tarde, como resultado do forrageamento pelas abelhas e outros visitantes florais.

As maiores intensidades de coleta de pólen coincidem com as temperaturas mais elevadas em ambos períodos.

Nos meses mais quentes verificou-se que a coleta de pólen foi mais intensa entre 40 e $79 \%$ de umidade relativa e na faixa de irradiação solar de 200 a $799 \mathrm{~W} / \mathrm{m}^{2}$. Diferentemente, durante os meses mais frios a coleta de pólen foi mais intensa entre 50 a $69 \%$ de umidade relativa e entre 300 e $499 \mathrm{~W} / \mathrm{m}^{2}$ de irradiação solar. O pólen foi coletado com maior intensidade na faixa de 18 a $19^{\circ} \mathrm{C}$, independentemente das estações do ano, tanto na postura quanto na diapausa. No entanto, os valores meteorológicos limitantes para as coletas de pólen mostraram-se mais amplos (Tab. I).

Tabela I. Valores mínimos e máximos dos parâmetros meteorológicos registrados durante coleta de pólen por $P$. saiqui, no período de outubro/1998 a setembro/1999.

\begin{tabular}{lcccc}
\hline Periodo & Irradiação solar $\left(\mathrm{W} / \mathrm{m}^{2}\right)$ & Temperatura $\left({ }^{\circ} \mathrm{C}\right)$ & Umidade relativa $(\%)$ & Vento $(\mathrm{m} / \mathrm{s})$ \\
\hline Postura & $20-879$ & $12,9-26,7$ & $42-107$ & $0-6,1$ \\
Diapausa & $107-432$ & $16,9-22,4$ & $37-76$ & $0-8,1$ \\
\hline
\end{tabular}

Durante a postura as análises de regressão simples (diária e sazonal) e múltipla (sazonal) indicaram que a umidade relativa, a irradiação solar e a temperatura influenciaram significativamente no forrageamento de pólen. Dentre esses, a umidade relativa destacou-se como o fator maior importância (Tabs II-V).

Tabela II. Análise (diária) da regressão simples realizada durante a postura da rainha, relacionando a influência dos parâmetros meteorológicos na coleta de pólen de $P$. saiqui. Coeficiente de correlação $(r)$; nivel de significância $(P)$ destacado em negrito $P<0,05$; entre parênteses a colônia analisada; não houve coleta de pólen (-).

\begin{tabular}{|c|c|c|c|c|c|c|c|c|}
\hline \multirow[t]{2}{*}{ Data } & \multicolumn{2}{|c|}{ Irradiação solar } & \multicolumn{2}{|c|}{ Temperatura } & \multicolumn{2}{|c|}{ Umidade relativa } & \multicolumn{2}{|c|}{ Vento } \\
\hline & $r$ & $\mathrm{P}$ & $r$ & $P$ & $r$ & $\mathrm{P}$ & $r$ & $\mathrm{P}$ \\
\hline $09 / X / 98(A)$ & 0,588 & 0,016 & 0,707 & 0,002 & $-0,751$ & 0,000 & $-0,015$ & 0,954 \\
\hline $09 / X / 98$ (D) & 0,721 & 0,001 & 0,635 & 0,008 & $-0,696$ & 0,002 & $-0,339$ & 0,197 \\
\hline $18 / X / 98(A)$ & 0,216 & 0,420 & 0,336 & 0.202 & $-0,436$ & 0,091 & -0.019 & 0,943 \\
\hline 18/X/98 (D) & - & - & - & - & - & - & - & - \\
\hline $19 / X I / 98(A)$ & 0,291 & 0,272 & 0,470 & 0,065 & $-0,520$ & 0,038 & 0,295 & 0,267 \\
\hline $19 / X I / 98$ (D) & 0,448 & 0,081 & 0,591 & 0,015 & $-0,644$ & 0,007 & $-0,209$ & 0,435 \\
\hline $24 / X I / 98(A)$ & 0,776 & 0,000 & 0,637 & 0,007 & $-0,636$ & 0,007 & 0,263 & 0,324 \\
\hline $24 / X 1 / 98$ (D) & 0,646 & 0,006 & 0,437 & 0,090 & $-0,571$ & 0,020 & 0,408 & 0,116 \\
\hline $03 / X I 1 / 98(A)$ & 0,584 & 0,017 & 0,377 & 0,149 & $-0,590$ & 0,016 & $-0,176$ & 0,513 \\
\hline 03/XII/98 (D) & 0,642 & 0,007 & 0,436 & 0,090 & $-0,662$ & 0,005 & $-0,137$ & 0,611 \\
\hline $21 / X I I / 98(A)$ & 0,706 & 0,002 & 0,757 & 0,000 & $-0,555$ & 0,025 & $-0,096$ & 0,723 \\
\hline $21 / X 11 / 98$ (D) & 0,807 & 0,000 & 0,763 & 0,000 & 0,513 & 0,041 & $-0,251$ & 0,347 \\
\hline
\end{tabular}

Entretanto, na diapausa, as análises de regressão simples (diária) e múltipla (sazonal), evidenciaram que principalmente a irradiação solar e a temperatura influenciaram significativamente na coleta de pólen. Esses dados corroboram com os resultados obtidos na análise de regressão simples (sazonal) (Tabs II-V). 
Com relação ao vento, as análises de regressão evidenciaram que, tanto durante a postura como na diapausa, este não exerceu influência significativa na coleta de pólen pelas abelhas (Tabs II-V).

Tabela III. Análise (diária) da regressão simples realizada durante diapausa, relacionando a influência dos parâmetros meteorológicos na coleta de pólen de $P$. saiqui. Coeficiente de correlação (r); nível de significância $(P)$ destacado em negrito $P<0,05$; entre parênteses a colônia analisada.

\begin{tabular}{|c|c|c|c|c|c|c|c|c|}
\hline \multirow[t]{2}{*}{ Data } & \multicolumn{2}{|c|}{ Irradiação solar } & \multicolumn{2}{|c|}{ Temperatura } & \multicolumn{2}{|c|}{ Umidade relativa } & \multicolumn{2}{|c|}{ Vento } \\
\hline & $r$ & $\mathrm{P}$ & $r$ & $P$ & $r$ & $P$ & $r$ & $P$ \\
\hline $17 N / 99(A)$ & 0,594 & 0,015 & 0,628 & 0,009 & $-0,548$ & 0,027 & $-0,221$ & 0,409 \\
\hline $17 N / 99$ (D) & 0,654 & 0,005 & 0,679 & 0,003 & 0,616 & 0,010 & 0,257 & 0,334 \\
\hline $26 / N 1 / 99(A)$ & 0,493 & 0,052 & 0,628 & 0,009 & $-0,394$ & 0,130 & $-0,020$ & 0,940 \\
\hline $26 / \mathrm{VI} / 99$ (D) & 0,577 & 0,019 & 0,613 & 0,011 & 0,348 & 0,185 & 0,079 & 0,768 \\
\hline $28 / \mathrm{NI} / 99(\mathrm{~A})$ & 0,504 & 0,046 & 0,639 & 0,007 & $-0,395$ & 0,129 & 0,158 & 0,556 \\
\hline $28 / \mathrm{NI} / 99$ (D) & 0,670 & 0,004 & 0,770 & 0,000 & $-0,380$ & 0,145 & 0,166 & 0,536 \\
\hline $12 / \mathrm{VII} / 99(\mathrm{~A})$ & 0,627 & 0,009 & 0,644 & 0,007 & $-0,611$ & 0,011 & 0,032 & 0,905 \\
\hline $12 / \mathrm{VI} / 99$ (D) & 0,423 & 0,101 & 0,659 & 0,005 & $-0,597$ & 0,014 & $-0,220$ & 0,411 \\
\hline $13 / \mathrm{VII} / 99(\mathrm{~A})$ & 0,504 & 0,046 & 0,718 & 0,001 & $-0,393$ & 0,131 & $-0,371$ & 0,156 \\
\hline 13/VII/99 (D) & 0,571 & 0,020 & 0,655 & 0,005 & $-0,488$ & 0,055 & 0,017 & 0,949 \\
\hline
\end{tabular}

Tabela IV. Análise de regressão simples do período de postura e diapausa (sazonal), relacionando a influência dos parâmetros meteorológicos na coleta de pólen de $P$. saiqui. Coeficiente de correlação (r); nível de significância $(P)$ destacado em negrito $P<0,05$; entre parênteses a colônia analisada.

\begin{tabular}{|c|c|c|c|c|c|c|c|c|}
\hline \multirow{2}{*}{ Período } & \multicolumn{2}{|c|}{ Irradiação solar } & \multicolumn{2}{|c|}{ Temperatura } & \multicolumn{2}{|c|}{ Umidade relativa } & \multicolumn{2}{|c|}{ Vento } \\
\hline & $r$ & $P$ & $r$ & $\mathrm{P}$ & $r$ & $P$ & $r$ & $\mathrm{P}$ \\
\hline Postura (A) & 0,461 & $2,190 \times 10^{-6}$ & 0,529 & $2,910 \times 10^{-8}$ & $-0,573$ & $9,950 \times 10^{-10}$ & 0,048 & 0,636 \\
\hline Postura (D) & 0,463 & $1,480 \times 10^{-5}$ & 0,584 & $1,260 \times 10^{-8}$ & $-0,604$ & $2,830 \times 10^{-9}$ & $-0,012$ & 0,912 \\
\hline Diapausa (A) & 0,322 & 0,003 & 0,218 & 0,052 & $-0,108$ & 0,338 & $-0,216$ & 0,054 \\
\hline Diapausa (D) & 0,547 & $1,450 \times 10^{-7}$ & 0,538 & $2,520 \times 10^{-7}$ & $-0,316$ & 0,004 & $-0,125$ & 0,266 \\
\hline
\end{tabular}

Tabela V. Análise múltipla (sazonal), durante o período de postura e diapausa, relacionando a influência dos parâmetros meteorológicos na coleta de pólen de $P$. saiqui. Nível de significância $(P)$, destacado em negrito $P<0,05$; entre parênteses a colônia analisada.

\begin{tabular}{ccccc}
\hline \multirow{2}{*}{ Período } & \multicolumn{4}{c}{ Nivel de significância (P) } \\
\cline { 2 - 5 } & Irradiação solar & Temperatura & Umidade relativa & Vento \\
\hline Postura (A) & 0 & 0 & 0 & 0,852 \\
Postura (D) & 0 & 0 & 0 & 0,698 \\
Diapausa (A) & 0 & 0 & 0 & 0,602 \\
Diapausa (D) & 0 & 0 & 0,232 & 0,467 \\
\hline
\end{tabular}

\section{Análise do pólen armazenado}

O pólen depositado em potes foi amostrado mensalmente, exceto nos meses de julho/1999 (Colônias E e F), abril/1999 e junho/1999 (Colônia E) quando não ocorreu armazenamento (Figs 3 e 4).

Revta bras. Zool. 19 (1): $289-300,2002$ 
SILVEIRA (1991) e BIESMEIJER et al. (1992) mencionam a importância, para as abelhas, do volume dos grãos de pólen armazenado. Porém, neste estudo adotou-se a sistemática da representação percentual dos tipos polínicos possibilitando comparar quantitativamente as preferências florais das abelhas.
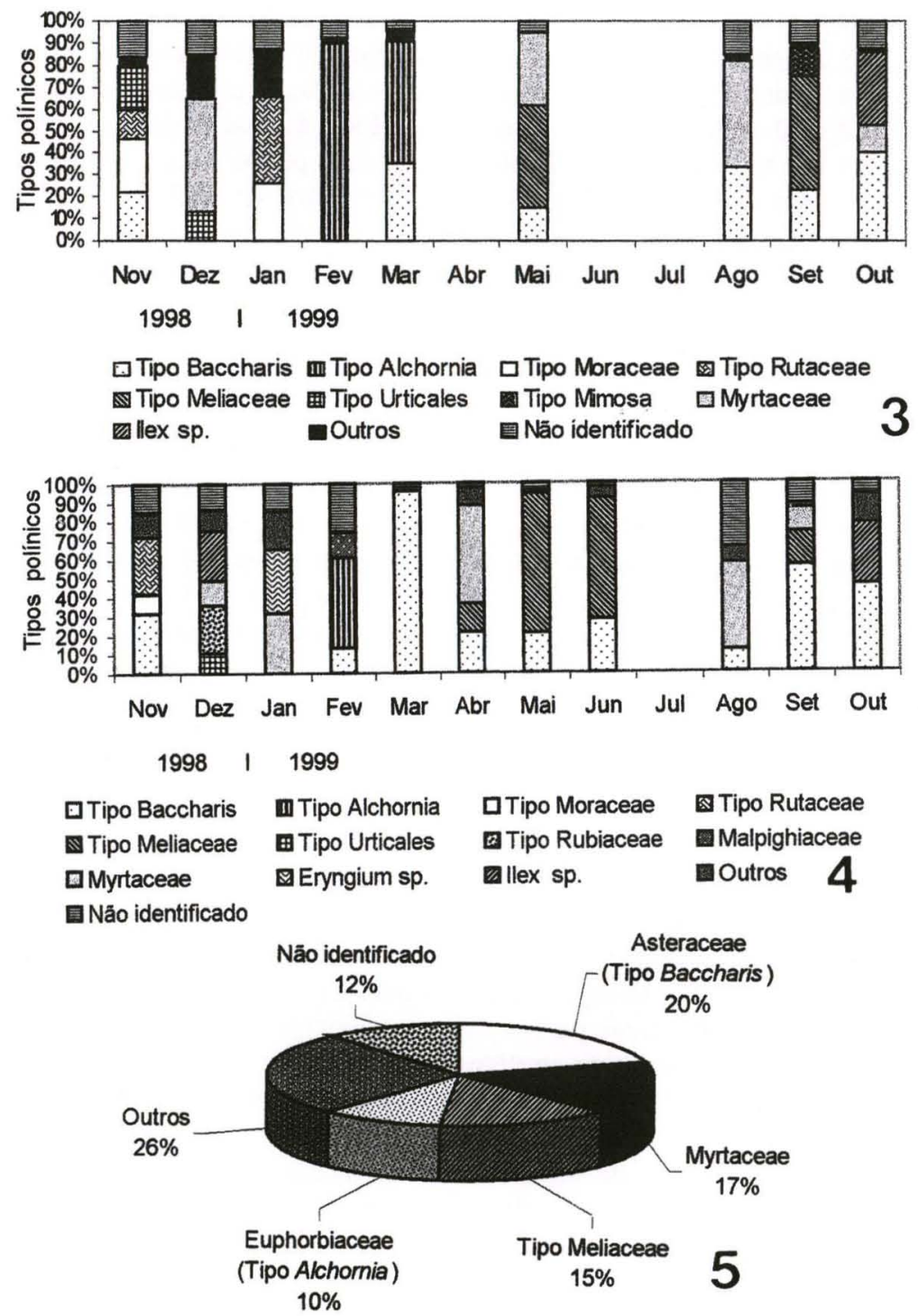

Figs 3-5. (3-4) Variação sazonal do pólen coletado ( $\geq 10 \%$ ) por $P$. saiqui: (3) Colônia E, (4) Colônia F; (5) representatividade das fontes polínicas encontradas nos potes de alimento de duas colônias de $P$. saiqui, ao longo do ano. 
A análise floral do pólen armazenado nas colônias, revelou diferenças marcantes quando comparadas com amostras coletadas em áreas urbanas. Em São Paulo, estudos sobre os recursos polínicos de $P$. saiqui, destacaram como fontes importantes de alimento, as seguintes famílias: Balsaminaceae, Euphorbiaceae, Moraceae, Myrtaceae e Arecaceae (KLEINERT-GIOvANNINI et al. 1987; IMPERATRIZ-FONSECA et al. 1989; PIRANI \& CORTOPASSI-LAURINO 1994). Convém mencionar que em ambientes urbanos freqüentemente se cultiva espécies exóticas usadas como plantas ornamentais, como é o caso de Archontophoenix cunninghamiana $\mathrm{H}$. Wendl. (Palmae) e Impatiens sultani Hook. S. (Balsaminaceae). Ambas florescem o ano todo, disponibilizando pólen e néctar permanentemente (IMPERATRIZ-FONSECA et al. 1989; PIRANI \& CORTOPASSI-LAURINO 1994).

Diferenças na dieta de $P$. saiqui podem ser atribuídas às influências da temperatura, umidade e $\mathrm{pH}$ do solo, que afetam o valor nutritivo do pólen. Consequentemente, o pólen de uma espécie pode diferir quimicamente quando coletado em locais diferentes (TODD \& BRETHERICK 1942). Além disso, a quantidade de pólen e de néctar varia entre as espécies, em uma mesma espécie e até mesmo entre flores de um mesmo indivíduo (RANTA \& LUNDBERG 1981). Estas variáveis podem ter sido determinantes para a escolha das fontes de pólen de $P$. saiqui, na região de estudo. A operárias coletaram principalmente grãos de pólen de Myrtaceae e dos tipos Alchornea (Euphorbiaceae), Meliaceae e Baccharis (Asteraceae) (Fig. 5).

O tipo Baccharis (Asteraceae) esteve presente em quase todas amostras e apresentou percentual relevante no espectro polínico total para ambas colônias. Nessa área encontram-se dezenove espécies de Baccharis, que florescem quase todo ano, exceto nos meses de junho e julho. Além disso, o período de floração pode variar de três semanas a três meses, dependendo da espécie. Baccharis spp. disponibilizam recursos alimentares por longos períodos, com floradas abundantes, sobrepostas e atrativas tanto para coleta de pólen como de néctar, e por isso, são importantes para manutenção de colônias de $P$. saiqui ao longo do ano (Birgit Hartes-Marques comunicação pessoal).

Entretanto, apesar desta fonte floral apresentar ampla distribuição sazonal, nem sempre foi intensamente explorada pelas abelhas. De outubro a dezembro, florescem dez espécies de Baccharis coincidindo com o período de maior diversidade de recursos florais (Birgit Hartes-Marques comunicação pessoal). Diante disso, verifica-se que $P$. saiqui, passa a explorar maior número de espécies vegetais, seja para procurar recursos mais atrativos ou para evitar a competição com outros insetos, uma vez que as flores de Baccharis spp. são freqüentemente visitadas por representantes de Coleoptera, Diptera e Vespoidea.

Dentre as espécies de Apidae, em especial as abelhas sem ferrão, são consideradas importantes agentes polinizadores de Baccharis spp. RAMALHO et al. (1990) ressaltam que flores de Asteraceae são consideradas as mais consistentes fontes de pólen e néctar do sul do Brasil, destacando-se como importantes recursos florais em vegetação aberta, freqüentemente resultante da ação antrópica, como ocorreu em parte da região de estudo.

Outra planta pioneira abundante na Floresta com Araucária, é a Mimosa scabrella (Leguminosae). Oferece néctar e pólen em abundância nos meses de 
setembro e outubro, sendo muito procurada por insetos, principalmente $P$. saqui e Apis mellifera Linnaeus, 1758 (Birgit Hartes-Marques comunicação pessoal). Apesar disso, esta fonte floral correspondeu a apenas $13 \%$ das coletas de pólen em setembro na colônia E (Fig. 3).

Em contrapartida, durante o inverno a oferta de alimento torna-se escassa e consequentemente a atividade forrageadora reduzida. Além disso, essa época coincide com o período de diapausa de $P$. saiqui, ou seja, período caracterizado por uma parada temporária na atividade reprodutiva da colônia (NECHOLS et al. 1999). FEWELL \& WINSTON (1992), estudando Apis mellifera, verificaram que o forrageamento de pólen é estimulado pela presença de cria na colônia, entretanto a ausência de imaturos não cessa a coleta de pólen.

As colônias de $P$. saiqui aprovisionaram poucos potes de pólen durante o inverno. Apesar dos recursos alimentares serem escassos e as condições meteorológicas nem sempre favoráveis à coleta de alimento, pólens do tipo Meliaceae foram representativos nas amostras de maio e junho (Figs 3 e 4).

Segundo RAMALHO et al. (1985), fontes com representatividade polínica de até $10 \%$ podem ser consideradas recursos de pouca atratividade, e devem corresponder a fontes potenciais ou secundárias. Estas, provavelmente complementariam as necessidades das colônias, bem como auxiliariam na manutenção do equilíbrio nutricional da dieta (Tab. VI).

Tabela VI. Fontes polínicas identificadas em potes de alimento $P$. saiqui, durante o período de novembro/1998 a outubro/1999, em São Francisco de Paula, Rio Grande do Sul. Pólen com representatividade $<10 \%(x)$; pólen com representatividade $\geq 10 \%$ no mínimo em um mês (xx).

\begin{tabular}{|c|c|c|c|}
\hline Família/Tipo & Tipo/gênero/espécie & Colônia E & Colônia F \\
\hline \multirow[t]{2}{*}{ Asteraceae } & Tipo Baccharis & $x x$ & $x x$ \\
\hline & Tipo Vernonia & $x$ & $x$ \\
\hline Apiaceae & Eryngyum sp. & $x$ & $x x$ \\
\hline Aquifoliaceae & Ilex sp. & $x x$ & $x x$ \\
\hline Cucurbitaceae & Tipo Cayaponia & $x$ & $x$ \\
\hline Euphorbiaceae & Tipo Alchornea & $x x$ & $x x$ \\
\hline Haloragaceae & Gunnera sp. & $x$ & $x$ \\
\hline Leguminosae & Tipo Mimosa & $x x$ & $x$ \\
\hline Loranthaceae & Phrygilanthus sp. & & $x$ \\
\hline Malpighiaceae & & & $x x$ \\
\hline Melastomataceae & Tipo Tibouchina & $x$ & $x$ \\
\hline Solanaceae & Tipo Solanum & $x$ & $x$ \\
\hline Tipo Meliaceae & & $x x$ & $x x$ \\
\hline Tipo Moraceae & & $x x$ & $x x$ \\
\hline Myrtaceae & & $x x$ & $x x$ \\
\hline Tipo Urticales & & $x x$ & $x x$ \\
\hline Tipo Rutaceae & & $x x$ & $x x$ \\
\hline Tipo Rubiaceae & & & $x x$ \\
\hline
\end{tabular}

Levando em consideração que $P$. saiqui é tipicamente encontrada em São Francisco de Paula, onde há grande diversidade vegetal, verificou-se dados semelhantes aos de outros autores, onde meliponíneos obtém grande parte do alimento em poucas famílias vegetais (IMPERATRIZ-FONSECA et al. 1984; KLEINERT-GIOVANNINI et al. 1987; RAMALHO 1990).

Revta bras. Zool. 19 (1): $289-300,2002$ 
Paralelamente, $P$. saiqui, apresentou flexibilidade na exploração de novas fontes florais. Em 26 de junho de 1999, junto à entrada do ninho, foi capturada uma operária de $P$. saiqui com uma polínia de Maxillaria picta Hook (Orchidaceae) no tórax. Dados da literatura mencionam que esta orquídea, em São Paulo é polinizada por outro meliponíneo muito comum, Trigona spinipes (Fabricius, 1793) (SINGER \& COCUCCI 1999).

Muitos meliponíneos são caracterizados como forrageadores oportunistas, coletando principalmente nas pétalas o pólen deixado por abelhas que realizam polinização por vibração. Em observações casuais, verificou-se que $P$. saiqui, visitou também espécies vegetais com anteras poricidas, a exemplo de Solanum variabile Mart. (Solanaceae). Vale a pena ressaltar que tais visitas foram restritas, e por isso, devem constituir recurso alimentar ocasional, revelando a capacidade desse meliponíneo em usufruir de fontes disponibilizadas para outras abelhas.

Em síntese, pode-se dizer, que a atividade de coleta de pólen pelas abelhas é reflexo da influência de parâmetros meteorológicos -temperatura, umidade relativa e irradiação solar - os quais influenciam também os ciclos diários e/ou sażonais de produção de pólen e de néctar (MICHENER 1974; FREE 1993). Indubitavelmente também fatores internos, a exemplo das necessidades das colônias em período de postura e de diapausa, são determinantes para o esforço de coleta de pólen (IMPERATRIZ-FONSECA et al. 1984; PIRANI \& CORTOPASSI-LAURINO 1994).

AGRADECIMENTOS. À Dra. Vera Imperatriz-Fonseca (USP) e Dra. Márcia Ribeiro (USP) pelo auxílio na metodologia. Ao MSc. Sérgio Hilário (USP) pelas sugestões e bibliografias cedidas. Ao Dr. Luiz Glock (PUCRS) e MSc. Walter Castro (PUCRS), pela orientação das análises estatísticas. Ao Dr. Paulo Neves (ULBRA) e à MSc. Suzane Hilgert Moreira (UNISINOS) pelo auxílio na identificação dos grãos de pólen. À Dra. Birgit Harter pela consulta à Palinoteca de Referência do LPB (PUCRS). À CAPES pela concessão da bolsa.

\section{REFERÊNCIAS BIBLIOGRÁFICAS}

ABSY, M.L. \& W.E. KeRR. 1977. Algumas plantas visitadas para obtenção de pólen por operárias de Melipona seminigra merrillae em Manaus. Acta Amazonica 7 (3): 309-315.

Bertoletti, J.J. \& M.B Teixeira. 1995. Centro de Pesquisas e Conservação da Natureza Pró-Mata. Termo de Referência. Divul. Mus. Ciênc. Tecnol. UBEA/PUCRS, Porto Alegre, 2: 1-47.

Biesmejer, J.C.; B. van Marwijk; K. van Deursen; W. Punt \& M.J. Sommeijer. 1992. Pollen sources for Apis mellifera L (Hym, Apidae) in Surinam, based on pollen grain volume estimates. Apidologie 23: $245-256$.

FAeGri, K. \& J. IVERSEN. 1989. Textbook of pollen analysis. London, Alden Press, $4^{\text {th }}$ ed., 328p.

FEWELL, J.H. \& M.L. WinStON. 1992. Colony state and regulation of pollen foraging in the honey bee, Apis mellifera L. Behav. Ecol. Sociobiol. 30: 387-393.

FREE, J.B. 1993. Insect pollination of crops. London, Academic Press, 684p.

FreE, J.B. \& I.H. Williams. 1977. The pollination of crops by bees. Bucharest, Apimondia Publ. House, p. 3-14.

GRONQUIST, A. 1981. An integrated system of classification of flowering plants. New York, Columbia Univ. Press, 126p.

Herreira, L.F. \& E. Urrego. 1996. Atlas de polen de plantas útiles y cultivadas de la Amazonia Colombiana. The Quartenary of Colombia 23. Botogá, Tropenbos Colombia, 464p. 
Hooghiemstra, H. 1984. Vegetation and climatic history of the High Plain of Bogotá, Colombia: a continuous record of the last 3,5 million years. Vaduz, Straless \& Cramer, 368p.

IBGE. 1986. Levantamento de Recursos Naturais. Rio de Janeiro, IBGE, Vol. 33, 796p.

Imperatriz-FonseCA, V.L.; A. Kleinert-Giovannini; M. Cortopassi-Laurino \& M. Ramalho. 1984. Hábitos de coleta de Tetragonisca angustula angustula Latreille (Hymenoptera, Apidae, Meliponinae). Bol. Zool. São Paulo 8: 115-131.

ImPERATRIZ-FonseCA, V.L.; A. KleinerT-Giovannini \& J.T. PiReS. 1985. Climate variation influence on the flight activity of Plebeia remota Homberg (Hymenoptera, Apidae, Meliponinae). Revta bras. Ent. 29 (3/4): 427-434.

IMPERATRIZ-FonseCA, V.L.; A. Kleinert-Giovannini \& M. RAmalho. 1989. Pollen harvest by eusocial bees in a non-natural community in Brazil. Jour. Trop. Ecol. 5: 239-242.

IwAmA, S. 1977. A influência de fatores climáticos na atividade externa de Tetragonisca angustula (Apidae, Meliponinae). Bol. Zool. São Paulo 2: 189-201.

Iwama, S. \& T. Melhem. 1979. The pollen spectrum of the honey of Tetragonisca angustula Latreille (Apidae, Meliponinae). Apidologie 10 (3): 275-295.

KERR, W.E. 1996. Biologia e manejo da tiúba: A abelha do Maranhão. São Luís, EDUFMA, 156p.

KLeinert-Giovannini, A. 1982. The influence of climatic factors on flight activity of Plebeia emerina Friese (Hymenoptera, Apidae, Meliponinae) in winter. Revta bras. Ent. 26 (1): 1-13.

Kleinert-Giovannini, A.; V.L. Imperatriz-Fonseca \& M. Ramalho. 1987. Explotation of floral resources by Plebeia saiqui Friese (Apidae, Meliponinae), p.156-157. In: J. EDER \& H. REMBOLD (Eds). Chemistry and Biology of Social Insects. Munique, Verlag J. Pepemy, 757p.

MiCHENER, C.D. 1974. The social behavior of the bees: a comparative study. Cambridge, Harvard Univ. Press, 404p.

Nechols, J.R; M.J. Tauber; C.A. Tauber; S. Masaki. 1999. Adaptations to hazardous seasonal conditions: dormancy, migration and polyphenism, p. 159-200. In: C.B. HUFFAKER \& A.P. Gutierrez (Eds). Ecological entomology. New York, John Wiley \& Sons, $2^{\text {nd }}$ ed., 756p.

Oliveira, M.A.C. 1973. Um método para a avaliação das atividades de vôo em Plebeia saiqui (Friese) (Hymenoptera, Apidae, Meliponinae). Bol. Zool. Biol. Mar., n.s., São Paulo, 30: 625-631.

Pirani, J.R. \& M. Cortopassi-Laurino. 1994. Flores e abelhas de São Paulo. São Paulo, Ed. Univ. São Paulo, $2^{\mathrm{a}}$ ed., 192p.

Ramalho, M. 1990. Foraging by stingless bees of the genus, Scaptotrigona (Apidae, Meliponinae). Jour. Apic. Res. 29 (2): 61-67.

Ramalho, M.; V.L. Imperatriz-FonseCa; A. Kleinert-Giovannini \& M. Cortopassi-Laurino. 1985. Exploration of resources by Plebeia remota (Holmberg) (Apidae, Meliponinae). Apidologie 16 (3): 307-330.

Ramalho, M.; A. KLeineRT-Giovannini \& V.L. ImperatRiz-FonseCA. 1990. Important bee plants for stingless bees (Melipona and Trigonini) and Africanized honeybees (Apis mellifera) in neotropical habitats: a review. Apidologie 21: 469-488.

Ramalho, M.; V.L. Imperatriz-Fonseca \& A. Kleinert-Giovannini. 1991. Ecologia nutricional de abelhas sociais, p. 225-252. In: A.R. PANIzZI \& J.R.P. PARRA (Eds). Ecologia nutricional de insetos e suas implicações no manejo de pragas. São Paulo, CNPQ, 359p.

RANTA, E. \& H. LUNDBERG. 1981. Food niche analyses of bumblebees: a comparison of three data collecting methods. Oikos 36: 12-16.

RouBIK, D.W. 1989. Ecology and natural history of tropical bees. New York, Cambridge Univ. Press, $514 \mathrm{p}$.

Roubik, D.W. \& J.E. Moreno. 1991. Pollen and spores of Barro Colorado Island. Monogr. Syst. Bot. Missouri Botanical Garden 36: 1-269.

Salgado-Labouriau, M.L. 1973. Contribuição à palinologia dos cerrados. Rio de Janeiro, Acad. Bras. Ciências, 291p.

SILVEIRA, F.A. 1991. Influence of pollen grain volume on the estimation of the relative importance of its source to bees. Apidologie 22: 495-502. 
Singer, R.B. \& A.A. CocucCI. 1999. Pollination mechanisms in four sympatric southern Brazilian epidendroideae orchids. Lindleyana 14 (1): 47-56.

Teixeira, M.B. \& A.B. Coura-Neto. 1986. Vegetação - As Regiões Fitoecológicas, sua Natureza e seus Recursos Econômicos. Estudo Fitogeográfico, p. 541-632. In: Levantamento de Recursos Naturais. 33. IBGE, Rio de Janeiro, 796p.

TodD, F.E. \& O. BRETHERICK. 1942. The composition of pollens. Jour. Econ. Entomol. 35 (3): 312-316.

VERGERON, P. 1964. Interprétation statisque des résultats en matière d'analyse pollinique des miels. Ann. Abeille 7 (4): 349-364.

Wilms, W; L. LutZ; A. Zillikens; B. Blochtein \& W. Engels. 1997. Bees and other recorded on flowering trees in a subtropical Araucaria Florest in southern Brazil. Stud. Neotrop. Fauna Environ. 32: 220-226.

Wittmann, D. \& M. Hoffmann. 1990. Bees of Rio Grande do Sul, southern Brazil (Insecta, Hymenoptera, Apoidea). Iheringia (70): 17-43.

Wodehouse, R.P. 1935. Pollen grains. Their structure, identification, and significance in science and medicine. New York, MacGraw-Hill Inc., 574p.

Recebido em 13.VII.2001; aceito em 06.III.2002. 